\title{
Constructible Authentic Representations: Designing Video Games that Enable Players to Utilize Knowledge Developed In-Game to Reason About Science
}

\author{
Nathan R. Holbert • Uri Wilensky
}

(C) Springer Science+Business Media Dordrecht 2014

\begin{abstract}
While video games have become a source of excitement for educational designers, creating informal game experiences that players can draw on when thinking and reasoning in non-game contexts has proved challenging. In this paper we present a design principle for creating educational video games that enables players to draw on knowledge resources gained in-game to reason about non-game phenomena. Games that incorporate this design principle, which we call constructible authentic representations, engage players in the construction of artifacts that are visually and epistemologically aligned to tools and representations utilized in the target domain. We illustrate this principle with a study of six children (ages 7-13) playing a racing video game of our own design. Players that struggled with a formal graphing task before playing the game showed improvement on the same task in post-game interviews creating qualitatively correct velocity versus time graph that incorporated key kinematic features such as moments of constant velocity and varying degrees of acceleration. An analysis of pre- and post-game clinical interviews also revealed that players more fluidly drew on a variety of knowledge resources when reasoning about the game, real world, and formal representations. We hypothesize that designing games to include constructible authentic representations may allow for the creation of educational video games that can survive in the non-school gaming ecosystem.
\end{abstract}

Keywords Video games $\cdot$ Design $\cdot$ Construction $\cdot$ Epistemology $\cdot$ Physics

\section{Introduction}

Children play a lot of video games. Due to their popularity, educational designers have made an effort to bring video games into the classroom. These educational games, designed to directly teach scientific concepts and practices, have met with moderate

N. R. Holbert (四) - U. Wilensky

Northwestern University, Walter Annenberg Hall, 2120 Campus Drive, Evanston, IL 60208, USA

e-mail: nholbert@u.northwestern.edu 
success in the classroom, but have not seen widespread use among youth outside of school. This gap between the uses of popular and educational game design offers both an opportunity and a challenge: Can we design video games that fit squarely into youth culture while simultaneously offering an opportunity for science learning? Building upon successful technology-rich play spaces and drawing from research on the role of intuition in scientific reasoning we have taken up this challenge by creating video games designed to embed difficult scientific concepts into gameplay.

While these games have been designed to fit into youth gaming culture, our expressed goal is to better understand how specific designs contribute to the use of game experiences for scientific reasoning. In this paper we present one such game and highlight a central design feature that enables players to utilize knowledge developed in-game to reason about non-game phenomena. We illustrate this principle with a study of six children (ages 7-13) playing this game and use an analysis of pre- and post-game interviews to illustrate how the use of building tools in-game provided players with knowledge resources for reasoning about related non-game problems.

Multiple studies indicate that video games have become a nearly universal activity among youth. One such study suggests that as many as $97 \%$ of all American teens (regardless of gender, age, or socioeconomic status) play video games on computers, consoles, or portable devices and $50 \%$ play games daily for an hour or more (Lenhart et al. 2008). The ubiquity of video games has made them a major focus of educational research. Over the past decade, this research has suggested that video games can serve as powerful spaces to teach critical thinking, can enable identity exploration and development, and can effectively spawn real-world communities (Gee 2003; Itō 2010; Squire 2006). And while research showing a positive potential for video games as vehicles for learning continues to grow, there exists a tension between games created solely for entertainment purposes and educational games designed to teach content first and highlight entertainment second (Itō 2008).

In an effort to prove to policymakers that the learning that occurs during gameplay aligns with educational standards, many studies of educational games have relied on traditional standardized measures to assess their impact. To ensure success on these measures and to encourage adoption of video games in the classroom, many educational game designers have simply embedded traditional classroom content and curriculum into game-like systems. Designers that break with this formula, and instead attempt to infuse educational content into quality game experiences, are often forced to build traditional content or classroom discussions around these games to ensure that players adequately reflect on how in-game experiences relate to assessment items (Clark and Martinez-Garza 2012). However, even with this concerted effort, educational video games designed for the classroom have yet to live up to their promise, showing only moderate success on directly equivalent assessment items and even weaker results on items assessing related concepts (Annetta 2008; Barab et al. 2007b; Clark et al. 2010).

Like our colleagues, we are still optimistic that video games can be vehicles for experimenting with science phenomena. However, rather than design science video games for the classroom, we have chosen to explore design principles that can transform popular video games-games that are typically played outside of the school context and remain consistent with youth gaming culture-into powerful educational experiences. With this in mind, we have created a prototype video game, FormulaT Racing (FTR) (Holbert and Wilensky 2010a) to be an archetype of the racing genre while providing a platform for experimentation with new representations and interaction mechanisms. Instead of trying to explicitly "teach" players embedded physics concepts, FTR is designed to tap into 
children's intuitive notions of motion in order to connect these intuitions to formal representations. FTR is not designed to enable players to become experts in kinematics; rather, it is designed so that players get a sense that experiences in the game are relevant to nongame motion experiences and that formal definitions and representations of kinematic content are relevant to game experiences.

Designing a video game that provides powerful opportunities for knowledge construction while staying true to youth gaming culture is a tall order. On the one hand, we know that overtly "educational" games or games embedded in traditional curriculum often fail to be taken up by students beyond the game. Along with Barendregt and Bekker (2011) our conjecture is that while such games are fun when compared to typical classroom fare, they cannot compete with popular video games in out-of-classroom contexts. On the other hand, informal theories developed when playing very popular games such as Angry Birds aren't utilized when facing identical "projectile problems" in a typical physics classroom.

To overcome the first problem, we adopt a framework that proposes educational games should be designed to look and feel like traditional video games, and refrain from adopting a didactic approach or obvious educational agenda (Annetta 2008). Doing so, however, brings the second problem to the foreground. This paper will primarily address the second problem by proposing the constructible authentic representations (CAR) design principle:

To promote the use of encountered content in both game and non-game contexts, gameplay should provide players with opportunities to engage in meaningful construction with components that integrate relevant concepts to create in-game representations that visually and epistemologically align with tools and representations utilized in the target domain.

In CAR games the building blocks used for construction-and the way in which the player interacts with these primitives-directly integrate the target content. When building primitives are combined into larger artifacts to achieve success in-game, the resulting construction is visually and epistemologically aligned with related tools and representations that exist and have meaning outside of the game. We hypothesize that interactions with these constructible authentic representations encourage players to see experiences ingame as relevant when thinking and reasoning in other, more formal domains that utilize related representations.

In the following sections we highlight the challenge of designing educational games that address these two problems and describe theoretical constructs highly relevant to CAR designs. In the second half of the paper, we present a study of six children (ages 7-13) playing FTR and show how players utilize in-game representations and tools to reason about kinematic phenomena to further illustrate the design behind, and value of, constructible authentic representations. Specifically we seek to answer the following research questions:

1. Do FTR players show improvement on non-game and domain specific tasks between pre- and post-game interviews?

2. Do players use FTR experiences to solve non-game and domain specific problems in the post-game interview?

\subsection{Educational Game Design}

With the growing enthusiasm for the educational potential of video games a large number of projects have sprung up attempting to capitalize on this popular and potentially powerful 
activity. These projects generally fall into one of two categories: "edutainment" games for the home and educational games designed for the classroom.

The classic MathBlaster series, and other similar edutainment games such as the Muncher series, are marked by a design that makes the educational content overtly explicit by "gamifying" traditional classroom instruction techniques using simple video game mechanics and external incentives. These games often split the gameplay and "content"such as having the player complete sums in between laser firing sessions as in MathBlaster-or combine the gameplay with the content in meaningless ways-such as "eating" only vowels in Word Munchers. While typically designed for the home, many such games have found their way into the classroom. In the end, such games might succeed in motivating players to complete many simple problems but the educational value is little more than that of flashcards. Despite this disconnect, some edutainment games have proven successful at increasing learner motivation and at achieving modest learning gains (Papastergiou 2009).

Alternately, a number of video games have been created to teach science content in the classroom. One such game, Quest Atlantis (Barab et al. 2005, 2007b) is a multi-user virtual environment modeled after extremely popular games such as World of Warcraft. Rather than completing quests by killing monsters, Quest Atlantis players embark on quests more in line with inquiry science instruction. Similarly, River City (Ketelhut et al. 2010) engaged students in a "participatory historical simulation" (p. 95) to evaluate the water quality of a virtual city. These designs provide an interesting gaming context for engaging in valuable scientific practices and allows for experiences that are often difficult to achieve in a brick and mortar classroom. However, both games rely heavily on text-based narratives to deliver content rather than game mechanics and the structure and content of game quests are highly similar to activities and lessons one might find in a reform science classroom.

Other games meant for the classroom attempt to blend science content directly with game mechanics. SURGE (Clark et al. 2010) is a puzzle game that forces players to interact with principles of Newtonian Mechanics to achieve in-game goals. In SURGE, players move a ship through narrow spaces and obstacles by applying small impulses to save cartoon "fuzzles". Other in-game challenges include flying the ship at constant velocity or launching it from a high ledge with the correct initial force to land safely on a small platform below. In contrast to Quest Atlantis, SURGE focuses on content-based learning, rather than process, by having players directly interact with the concepts of interest.

An interesting variation on these educational games designed for the classroom is the use of popular commercial games wrapped in curriculum. A good example of such a design is Squire and Barab's (2004) use of Civilization III in history classes. Here players were encouraged to use the game mechanics to "replay history." In replaying history players were encouraged to reflect on possible mechanisms and causes for major historical events (such as the colonization of the Americas) and given the opportunity to imagine, and then to test, how things might have gone differently. Other examples of similar projects include the recently launched "Learn with Portals" initiative, where children use the Portal 2 level designer to participate in activities around physics, mathematics, and game design (Valve Corporation 2013), and a computational thinking curriculum that uses Angry Birds to teach projectile motion (Behesthi et al. 2013).

These games and related studies provided a rich set of examples that have influenced and shaped educational game design. However, findings from these games have been both encouraging and disappointing. Many such games are often successful in developing nonconceptual constructs such as engagement (Squire and Barab 2004) and identity development 
(Gee 2007). And while most of these games show modest gains on directly equivalent conceptual problems, in many cases, players fail to show gains on problems of related phenomena (Annetta 2008; Barab et al. 2007a; Clark and Martinez-Garza 2012). For example, players of SURGE showed gains on Force Concept Inventory items that matched ingame problems but failed to show gains on other, related FCI problems (Clark and MartinezGarza 2012; Clark et al. 2011). Likewise, in one implementation of Quest Atlantis, students showed gains on items chosen to directly align with the embedded curriculum but failed to make significant gains on "distal" items of the chosen standards (Barab et al. 2007a, b).

While we applaud much of the work that falls into these categories, there is an untapped potential for the design of games that meet player's expectations of play and thrive in the gaming ecosystem, while also providing a space for the exploration and experimentation of encountered science content. To achieve this goal, we put forward the constructible authentic representations principle.

\subsection{Theoretical Foundations}

Designers interested in creating educationally relevant video games must wrestle with the tradeoff of making the learning goals explicit versus foregrounding the game rules and narrative and fantasy (Collins 1995). An overemphasis on learning goals often results in an experience that feels more behaviorist in nature-drill and practice infused with animation and sound (Itō 2008) and as such, many beneficial aspects of games are lost. In contrast, by focusing solely on a game system, the embedded content that the researcher intends the player to experience may become clouded (Clark et al. 2011). The game may meet player expectations as an enjoyable experience, but is not seen as relevant in formal learning contexts (Itō 2008).

We propose that this inability to draw on game knowledge in formal contexts may be due to incongruent epistemological framing (Hammer et al. 2005) by players. In education research, the term "epistemology" is often used to describe an individual's beliefs about the form and type of knowledge relevant in a domain or context. Drawing on the theory of knowledge-inpieces (diSessa 1993), which suggests that cognition is emergent from the activation of a large number of fine-grained, highly distributed, context-sensitive primitive elements, Hammer et al. (2005) suggest that individual epistemologies change in-the-moment as various cognitive resources are activated. They go on to propose the idea of epistemological framing and define it as, "Phenomenologically, a set of expectations an individual has about the situation in which she finds herself that affect what she notices and how she thinks to act" (p. 98). In this model, the ability to recall or apply knowledge in a given context is affected by both the epistemological framing of the learning situation as well as the framing of the recall situation. In other words, when playing games where the educational content is implicit, players may not even consider that these experiences could have use elsewhere. The challenge, then, is to balance game rules and narrative with learning goals in such a way as to enable players to see in-game experiences as applicable when thinking and reasoning in non-game contexts. In this work we propose that this balance can be achieved by designing games to include constructible authentic representations.

Constructible authentic representations is a design principle meant to encourage the player to connect seemingly disparate knowledge resources making it more likely that game experiences will be co-activated with formal representations in non-game contexts. In this way players might see knowledge acquired during play as relevant beyond the game space. Games that include constructible authentic representations engage the player in construction with components, or primitives, that themselves integrate relevant conceptual 
resources and relationships between resources. Furthermore, artifacts constructed with these primitives are authentic, in that they visually and epistemologically align with related tools and artifacts utilized in the target domain. These authentic artifacts not only look like those utilized in the target domain, but the nature of the in-game artifacts also match the meaning and use of the target tools and representations. The ability to use these uniquely constructed representations (that visually and epistemologically match representations one might confront in formal learning contexts) to achieve in-game goals alters the individual's relationship to the representations, imbuing them with meaning and value (Pratt and Noss 2010). Such representations are no longer just "for school" but are now a useful tool for achieving personally meaningful goals. In short, CAR relies on three things: (1) opportunities for meaningful construction, (2) construction tools and primitives that integrate the concept of interest, and (3) visual and epistemological alignment between artifacts constructed by players and tools and representations central to the target domain.

CAR is informed by a constructivist view of cognition and rooted within the constructionist design paradigm. In line with models of cognition that describe human thought as an emergent phenomenon of many small sub-conceptual networked structures (diSessa 1993; Minsky 1986), constructionism proposes that the building of knowledge structures happens most effectively when "the learner is consciously engaged in building a public entity" (Papert and Harel 1991, p. 2). A constructionist approach facilitates knowledge system refinement by providing a space where individuals have the freedom to build in their own style to create something of personal meaning.

In CAR games, the tools and primitives used in construction integrate the concepts the designer intends the player to explore. This aspect of CAR builds upon the principle of conceptual integration (Cheng 2011; Clark et al. 2011; Habgood and Ainsworth 2011; Kafai 1996). Proponents of conceptual integration claim that the mode of interaction activates and refines in the child the same set of conceptual resources that would be activated when reasoning about some target real-world phenomenon (Fig. 1). This is possible because in a conceptually integrated design, the way the player interacts with the game IS the concept of interest (Clark et al. 2011; Habgood and Ainsworth 2011).

Habgood and Ainsworth (2011) created Zombie Division, a game designed to teach young children fractions, to illustrate and evaluate the value of a conceptually integrated design (they use the term intrinsic integration). In Zombie Division the various modes of attack available to the player embody the fractions of interest. For example, players using a sword effectively "divide" the zombie in two while punching using a five-fingered glove divides the zombie by five. Clark et al. (2011) provide another example of conceptual integration in SURGE. Here, players use impulses and moments of constant, positive, and negative acceleration to navigate a spaceship through a maze. In our own FTR (Holbert and Wilensky 2010a), which we will discuss in more detail in this paper, players roll motion-sensitive controls forward and backward to apply positive and negative acceleration to a racecar to complete various challenges. In this way, controller manipulation allows players to directly explore key kinematic concepts.

Unfortunately, players often fail to apply ideas learned in conceptually integrated games to non-game problems. Clark and Martinez-Garza (2012) hypothesize, "Though playing a conceptually-integrated game engages the player constantly in the targeted relationships, the player may never articulate or even identify those relationships." While subtlety is a strength of conceptually integrated games, this subtlety can also lead to inadequate player reflection. For this reason conceptually integrated games often require additional instruction or directed reflection to be effective. This directed reflection might take the form of in-game "prediction and explanation" activities (Clark and Martinez-Garza 2012) or teacher-led 


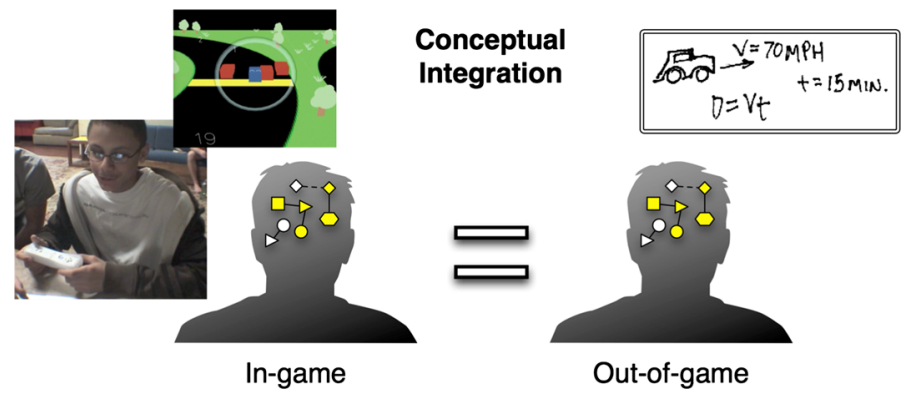

Fig. 1 In conceptually integrated games, interactions with the game mechanics develop and refine knowledge resources that match those utilized in the target domain

curriculum that connect in-game experiences to formal instruction (Boucher-Genesse et al. 2011; Robertson and Howells 2008). Unfortunately such solutions are inappropriate for games designed to be played informally. The CAR principle is an effort to overcome this shortcoming by implicitly connecting constructed artifacts (artifacts built using conceptually integrated primitives) to representations and tools easily recognizable in the target domain.

Constructed artifacts in CAR designs are meant to "stand between the concrete/ manipulable and the formal/abstract" (Noss and Hoyles 1996, p. 64; Wilensky 1991), much like Papert's (1980) most famous contribution to education research, the LOGO Turtle. In the programming language LOGO, the Turtle embodied the user's sense of motion and mathematics in the real world as well as the rules and features of formal mathematics. Papert (1980) suggests, "children can identify with the Turtle and are thus able to bring their knowledge about their bodies and how they move into the work of learning formal geometry" (p. 56). For example, by "playing turtle" the child can actually feel the appropriate moves necessary to walk out a square. In this way the idea of "squareness" isn't relegated to some abstract formula or set of rules and heuristics. It is instead a very real, and incredibly obvious thing-so obvious in fact that children sometimes have a difficult time using words to explain it! And because the words of the Turtle match the language of the child, creating a square, a triangle, or even a circle, is as simple as telling a friend how to move around in a room.

And yet, while playing turtle feels natural, and the language used to command the Turtle matches the language a child uses to describe her own motion in the world, the ideas enacted by the Turtle, and representations created by the Turtle, are very much mathematical. Whether constructing a house, a spiral, or a complex animation, the structures created by LOGO users embed highly formal rules and look remarkably like constructions one might find in the formal practices of engineers or computer animators. Pratt and Noss (2010) describe the Turtle as "auto-expressive" and claim, "What the turtle graphics commands like FD 50 manage to do is to combine computation-it will do something potentially useful when run-with representation-what it will do can be predicted from the representation" (p. 96). In this way creating with the Turtle not only allows the user to draw on her own sense of navigation in the world, but also to create artifacts that embody the relevant relationships and have value beyond the computer screen they are constructed on. Furthermore, because the mathematical ideas and relationships that are part of the LOGO language help the child to construct an artifact that is personally meaningful, the children's relationship to this mathematics changes. Now these ideas are no longer just 
"school" ideas, they are useful tools for achieving one's goals - the representations and embedded ideas have acquired utility (Pratt and Noss 2010). Just as the Turtle acts as a transitional object between intuition and formalizations, the primitives used for construction in CAR games are designed to tie directly into player's embodied way of reasoning about the world and artifacts constructed with these primitives embed concepts and processes of the target domain (Fig. 2).

While video games designed to include constructible authentic representations are rare, constructionist software often appear to utilize this design principle. Bamberger's (1996) Impromptu music software includes important representations to help users come to a better understanding of ideas like rhythm, but final constructions lead to representations and compositions recognizable by any musician. The programming environment Alice (Cooper et al. 2000; Kelleher and Pausch 2006) provides a space where users can experiment with object-oriented programming and quickly create professional looking animations. However, while Alice is tile-based, "instructions correspond to standard statements in a production oriented programming language, such as Java, $\mathrm{C}++$, and C\#" (The Alice Team n.d.). In all cases the work done by the constructed artifact, and the look and feel of the artifact, are congruent to the practice and forms of the target domain.

In our own game design work, CAR has been central. In Particles! (Holbert and Wilensky 2012), a platformer game for exploring the particulate nature of matter, players create in-game blocks with different physical properties by designing molecular-level structures. To do this, players bond many atoms together into larger structures that will serve as a template for all molecules that make up these new blocks (Fig. 3). This task involves meaningful construction as players are free to design any structure they wish, each leading to interesting emergent properties. Furthermore, the way the player creates these new blocks IS the target concept: namely bonding atoms together into molecular structures that lead to emergent physical properties. Finally, the representations the player interacts with in-game visually align with the ball-and-stick models used to represent atoms and molecules in the formal domain. Likewise, the structures players create in-game align with real molecular structures central to many interesting materials and properties observed in the real world.

In FTR, players use motion-sensitive controls to accelerate points up and down a y-axis to ultimately construct velocity-versus-time graphs that the player car will then utilize when driving around the track. Creating the graph involves meaningful construction in that players manipulate the points to create an artifact that will help them to either succeed or fail in their race around the track. The way players construct the graph integrates the target concept - the relationship between acceleration and velocity-as players accelerate graph points along the $y$-axis in the same way they accelerate their racecar around the track. Finally, the player-created graph is authentic in that it not only looks like a typical velocity-versus-time graph, but also has the same meaning and value as graphs encountered in both formal and real world contexts.

The above examples suggest that a variety of game types and content areas can be explored in constructible authentic representations designs. However, it is reasonable to wonder if all educational games or content domains naturally lend themselves to such a design. Furthermore, will CAR games be appropriate for learners of all ages? Or does the very nature of what we have described as "authentic representations" require that the learner have some prior knowledge or experience in the target domain? While we will not answer these questions directly in this paper, the findings from the study that follows will provide some insight into these issues and offer direction for future work which we will revisit in Sect. 4. 


\section{Constructible Authentic}

Representations

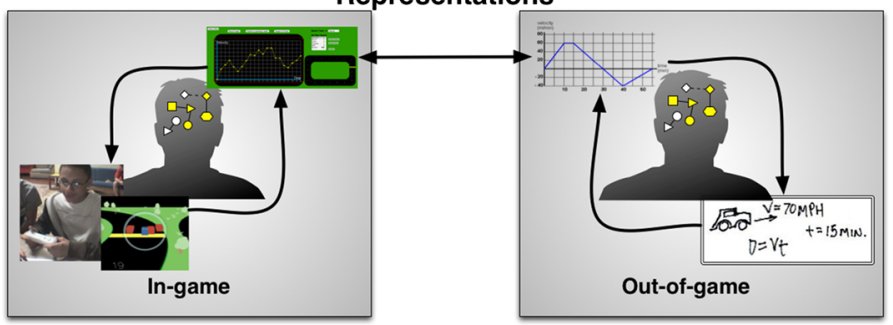

Fig. 2 In CAR games, conceptually integrated building primitives are used to construct artifacts central to gameplay. The resulting artifacts are also visually and epistemologically aligned to key tools and constructs utilized in the target domain. This alignment encourages players to see knowledge resources gained and developed in-game as relevant to the target domain and useful for achieving personally meaningful goals

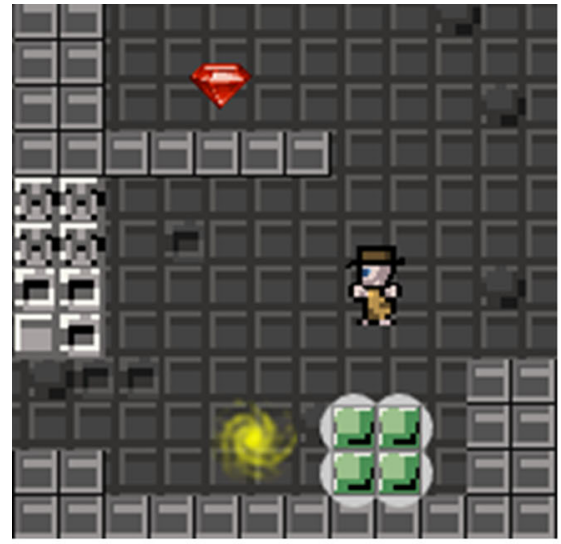

(a)

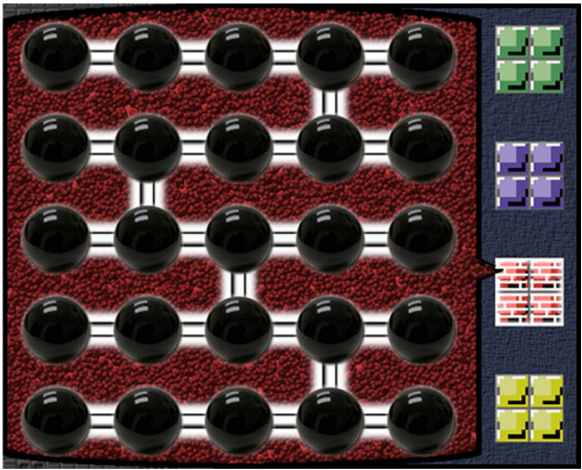

(b)

Fig. 3 In Particles! players design new blocks to be added to the game world by opening up a tool called the "atomizer." In the atomizer, players add and arrange bonds between atoms to create molecular-level structures (b). As new blocks are added to the game world a variety of physical properties emerge due the interaction of these player-created structures (a). Here the player has created a block with some bounce by cross-linking chains of atoms

Previously, we identified two challenges to educational game design: (1) designing games that align with youth gaming culture, and (2) building game experiences that players see as relevant to problem-solving in non-game contexts. In this section, we have outlined the core theoretical underpinnings of the constructible authentic representations design principle. While some educational games might be designed to overcome one of these two challenges, we propose that constructible authentic representations are uniquely capable of overcoming both design problems.

\section{Method}

In the remainder of the paper, we present a study of FTR intended to explore the value of designing games to include constructible authentic representations. Eleven players aged 
7-13 were recruited from various informal organizations throughout a large Midwestern US city. All participants were self-described "gamers" and six could be described as "expert" gamers in that they spent a large amount of time playing video games weekly. Of those six expert gamers two also considered themselves game designers as they had each made multiple games using Scratch (Resnick et al. 2009).

During recruitment players were told they would be helping us to evaluate a new game we were developing. This was done to encourage players to frame the interview and game playing as being about design and evaluation rather than a formal school-like task. After recruitment, pre-game interviews were conducted with participants to establish a baseline, which would indicate their conceptual knowledge of relevant content before playing the prototype game. Furthermore, because all participants considered themselves gamers, this baseline is also indicative of the knowledge players may have gained from previous gaming experiences.

About a week later participants played the game over two one hour play sessions. These game play sessions were generally conducted two to three days apart though this varied depending on participant schedules. Two to seven days after the second game playing session, a post-game interview with the player was conducted using the same protocol as the pre-game interview. ${ }^{1}$ Of the eleven children that completed the pre-game interview, five participants dropped out of the study due to scheduling conflicts before having the opportunity to play the game. The remaining six completed the pre-game and post-game interview playing the same version of the game.

Interviews and gameplay were conducted exclusively in informal settings, either in participant homes or the after-school program they attend, to remain consistent with typical video game playing contexts and to increase ecological validity (Brofenbrenner 1979). The pre-game interview itself was a semi-structured clinical interview centered around three main question groups and follow-up probes initiated by the interviewer as necessary (Ginsberg 1997; Piaget 1929). The interview began with warm-up questions about how often participants play games, what genre of games they play, and what specific racing games they have played. The three main question groups were:

1. Describing the motion of a car at a stoplight right after the light turns green.

2. Describing the motion of the car at a constant velocity as indicated by the needle of a speedometer.

3. Graphing the motion of a car based on the movement of the needle of a speedometer.

The questions themselves utilized props such as a toy car for the player to demonstrate answers, a paper speedometer with a movable needle for the interviewer to indicate various speeds in question groups two and three, and a blank graph with $\mathrm{x}$ - and $\mathrm{y}$ - axes labeled "velocity" and "time" for question group 3.

While the first two question groups focused on real-world examples of acceleration and velocity and were intended to probe participants' intuitive knowledge about motion, the third question group was designed to be a domain specific task to engage participants in reasoning with more formal concepts and representations. In the graphing task, participants are shown a paper speedometer (the term "speedometer" was never used by the interviewer, though most participants identified it as such) with a movable needle. While the interviewer slowly manipulates the speedometer needle, participants are asked to "make a graph describing what I am doing with this meter." This is done on a piece of graphing

1 Warm-up questions centered on feedback on the game rather than on commercial games participants have played. 
paper with the $\mathrm{x}$ - and y-axes pre-labeled "Time" and "Velocity" (the interviewer ensures all participants are familiar with the term "velocity" ahead of time, and if they are not, informs them that it is "kind of like speed"2).

Using methods described by Russ et al. (2012), we analyzed video data and transcripts of the interviews to look for moments where participant behavior and language indicate a shift in how he frames the interview activity. A coding scheme was developed bottom-up from multiple passes of the data though the grain-size of individual codes was informed using a framework developed by Hammer and Elby (2002). Graphs created for the third question group were analyzed (along with video data of graph construction) for important conceptual features, such as changing acceleration, as well as representation features, such as lines and points. Graphs were considered "qualitatively correct" if the participant produced a line graph that utilized both axes and showed changing velocity over time though all graphs where analyzed regardless of "correctness."

There are three main phases of FTR: skill development, racing, and constructing (a more detailed description of the design of FTR can be found in Holbert and Wilensky 2010b, 2011). The first two phases of FTR play like a traditional racing game, where the player must either complete various skill challenges or race around a track against competitor cars by accelerating and decelerating his car using a motion sensitive controller (in this study, players used a Nintendo Wiimote). Traditionally racing video games have provided kinematic feedback to the player through visual cues such as the passing background, haptic cues such as the controller, and numerical cues such as the speedometer. FTR leaves the traditional "passing background" visual cue, but adds a new "color-trails" representational cue. In this cue velocity is represented by a color trail left by the player vehicle that changes as the player car's velocity changes. While the color-trails are discrete (color changes are not gradual and occur once the player has passed a threshold speed) they provide a means to connect one's changing speed to the structure of the track. By observing the locations of "slow colors" relative to "fast colors," players are able to more easily see how their speed changed relative to track features. To continue to support this connection the player is given a bird's eye view of the total track, including their leftbehind color-trails, after they successfully complete a race. This very simple, but important representation allows players to consider the track as a series of fast and slow areas which will serve as both a scaffold as well as a building primitive for the interpretation and construction of more challenging representations.

Because our observations of children playing popular commercial racing games suggested players rarely attended to provided speedometers (Holbert 2009), a velocity-versustime graph that highlights changing speed was used instead. This formal kinematic representation serves two important goals. First, as a widely used representation, the velocityversus-time graph acts as an anchor to formal knowledge within the informal game-space. This mixing of formal representations and gameplay encourages the player to see gameknowledge as relevant across contexts. Since the player often identifies as an expert in gaming contexts, the application of this game-knowledge to non-game spaces is extremely powerful. Second, rather than a speedometer, which highlights instantaneous speed, a velocity-versus-time graph foregrounds changing velocity, or acceleration. Though acceleration has been shown to be a challenging concept for children to understand (Tasar 2010; Trowbridge and McDermott 1981), we believe that it is a powerful idea that provides

\footnotetext{
2 The speedometer does in fact measure speed rather that velocity. We chose to use the term "velocity" in the game and in the graphing task because of its alignment with formal terminology that may be used in the classroom.
} 
an entry point to many other difficult and useful concepts (Papert and Harel 1991; Papert 1980, 2000).

While these representations serve to foreground various kinematic principles, opportunities to allow learners to actively construct with these representations are central to providing opportunities for conceptual change. These constructionist designs (Papert and Harel 1991; Papert 1980) are inspired by work examining expert and novice embodied interactions with formal graphic representations (diSessa et al. 1991; Levy and Wilensky 2009; Nemirovsky et al. 1998; Roschelle et al. 2000; Sherin 2000; Trowbridge 1989; Wilensky and Reisman 2006; Wilkerson-Jerde and Wilensky 2010) and are intimately connected to previously discussed controls and visual cues but are not explicitly introduced until the third phase of the game. In this "pit boss level," rather than drive the car directly, the player constructs personal notions of motion by building a race plan in either a "painting" or "graphing" mode.

In the "painting" mode of the construction phase the player uses colors representing various velocities and corresponding to the previously seen color-trails to paint sections of the track. In this level, players are confronted with a birds-eye view of the entire track (Fig. 4). To paint the track, players select a color (which corresponds to a velocity) and paint the track by clicking and drawing with the mouse. Once the player is done painting the entire track, the car begins the race, changing velocity based on the color being driven over. If the player car is at a velocity lower than the painted section of the track, it slowly accelerates until it reaches the target speed. If the car is above the painted speed, it decelerates. The player is free to paint the track in any way they choose, however, because each color corresponds to a particular target velocity, and the car's ability to turn is impacted by its current velocity, the choices made in painting the track determine whether or not the car will successfully complete the race. In addition, the car must complete the track within a specified time limit. This ensures players cannot simply paint the entire track a slow color and instead forces them to experiment with different velocities in conjunction with different track features and to strategically plan their painting designs to achieve their goals.

After successfully completing the three included tracks by painting, the player then begins the second stage of the pit boss level. In this stage the player constructs a velocityversus-time graph that the car will then use to drive around the track. Using numbered markers distributed throughout the track, the player adds nodes to a graph to indicate the speed the vehicle should be traveling at that point (Fig. 5). However, rather than simply "clicking" to add a point at the chosen velocity, the player creates a point along the x-axis and then "accelerates" the point up and down the y-axis using the motion-sensitive controller. The controller used is the same the player has become familiar with in the previous racing phases of the game to accelerate his car around the track. By rolling the controller forward or backward, the player can add positive or negative acceleration to each point until he is satisfied with its location on the y-axis. Like the painting stage, the player must accelerate points on a graph over the course of the entire race, rather than accelerating the car in-the-moment. Once the graph is constructed, the car "downloads" the data and drives around the track according to the player-constructed graph. In this way the player directly connects the intuitive feeling of acceleration to formal graphic representations and can also explore how varying graphic features, such as sharp drops or plateaus in velocity correspond to particular track features.

In both the painting and graphing phases of the pit boss level, the player has the opportunity to make small or drastic changes to their constructions after the car has either crashed or made it around the track successfully. In previous work we have analyzed the 


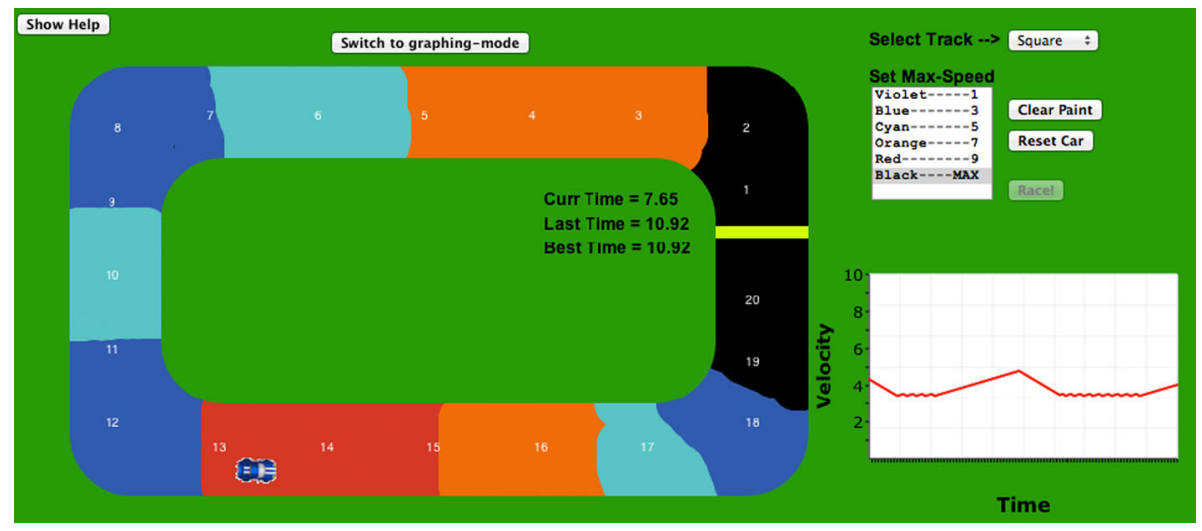

Fig. 4 Players paint the track using colors corresponding to different velocities. When the car drives over the painted colors, it accelerates and decelerates until its velocity matches that of the painted color

systematic changes players made and found that players engage in sophisticated debugging of constructions and many even utilize complex repeating patterns, or "procedures," as they work to come up with a winning construction (Holbert and Wilensky 2011).

In what way does FTR utilize constructible authentic representations? The racing phase of FTR directly instantiates two CAR components: conceptual integration and authentic representations. Recall that the goal of FTR is to help players make sense of the relationship between acceleration and velocity. The way the player uses the motion sensitive controller to accelerate their car, and the changing color trails left behind, directly integrate these kinematic concepts. Furthermore, as the player drives, a velocity versus time graph is dynamically created to replace the more traditional speedometer. This authentic representation highlights changing speed rather than instantaneous speed.

The construction phases of the game incorporate all three aspects of the CAR principle. In the drive-by-paint phase, the player utilizes the colors observed in the changing color trails (which integrates target kinematic ideas) to construct a complete representation of the race. In the drive-by-graph phase, players use the embodied controller to accelerate points up and down the y-axis until they create a complete graph that will be used to drive the car. In both cases the construction process integrates the target kinematic concepts and the completed artifact has important meaning in the context of the game. In the case of the drive-by-graph mode, this completed artifact also visually and epistemologically aligns with the line graph representation central in the practice of physics.

In the remainder of this paper, we present evidence from pre- and post-game interviews and the graphing task that indicates players are drawing on experiences in-game to reason about non-game phenomena and tasks. We argue that these changes are due to interactions with the constructible authentic representations utilized throughout FTR.

\section{Results}

Pre- and post-game graphs created by participants reveal that while many participants struggled in the pre-game graphing task (only three of eleven participants created qualitatively correct graphs during the pre-game interview), nearly all participants (five of the 


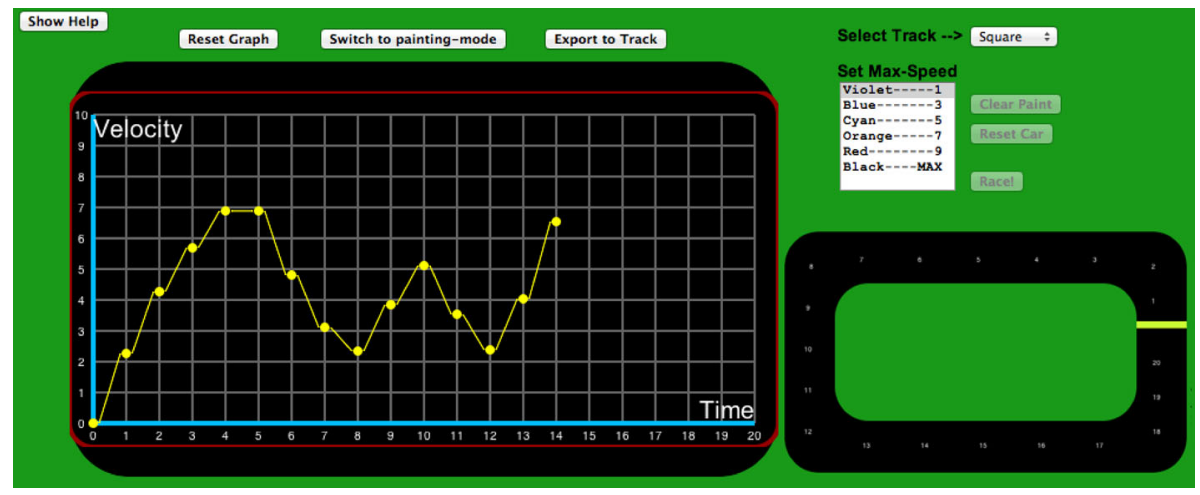

Fig. 5 In the graph construction phase, players accelerate points up and down the y-axis of a graph by rolling a motion sensitive controller forward (for positive acceleration) and backward (for negative acceleration)

six that played the game and completed post-game interviews) were able to create qualitatively correct graphs during the post-game graphing task despite the lack of any explicit instruction on graphing. Furthermore, the analysis of pre- and post-interviews below reveals that players utilized in-game experiences to reason about formal graph representations and other kinematic phenomena.

\subsection{Pre-game Interviews and Graphing}

In the pre-game interview, most participants struggled to produce a graph of the changes being made to the speedometer. Most graphs constructed by participants in the pre-game interview were unlike those formally utilized by the physics and education communities. In one common pre-game graph (five of eleven), players utilized the pencil as if it were the actual car being described by the changing speedometer. In other words, while the researcher increased the speed on the speedometer, the participant would move his pencil across the paper faster, and when the speedometer was moved to a slower speed, the participant slowed his pencil down (Fig. 6).

Five participants completed graphs that utilized both the $y$ - and $x$-axis. Two of these graphs were bar chart-like in nature and lacked features common in most velocity-versustime graphs (such as showing variations in both velocity as well as acceleration). The remaining three graphs were more traditional line graphs. However, on closer inspection of the line graphs it becomes apparent that two of these three graphs only indicate the velocity the car is traveling at a given moment and do not indicate variations in acceleration. One of these graphs, created by John (Fig. 7), shows a steady increase in velocity, then an area of constant velocity, and finally a steady decrease in velocity (both areas of increasing and decreasing velocity occur at the same rate). When asked about this graph John states simply, "it's like...the line is increasing, and then it stays the same, and then it's decreasing." John no doubt has some competence in graphing, but the representation created, and the description offered lacked features that might indicate a deeper understanding of the potential of the form.

The way in which participants construct a formal velocity versus time graph from movements of a needle on a speedometer during the interview is intimately connected to the participants' epistemological framing of the task. Participants framed the pre-game 


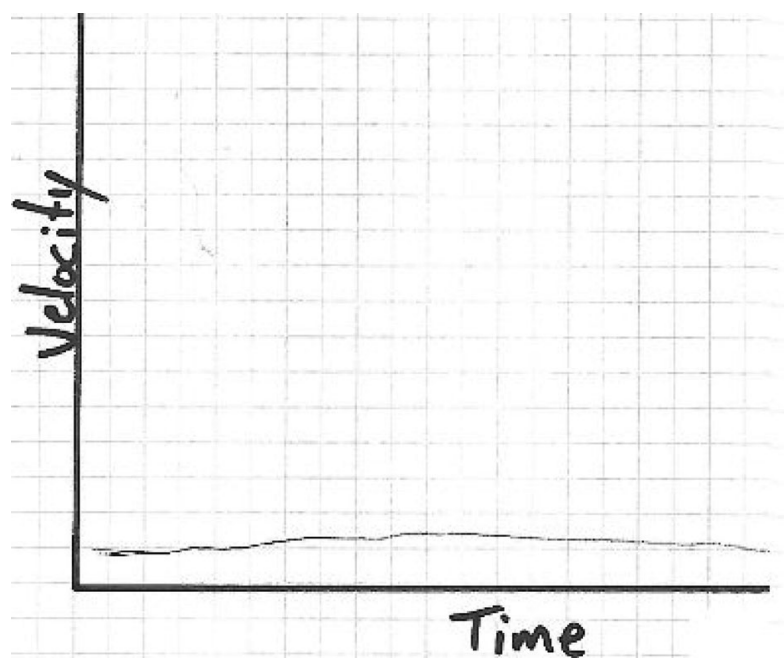

Fig. 6 In the common pre-game pencil-as-car graph, the participant accelerates his pencil along with the changes to the speedometer as if the pencil is the car

interview in a variety of ways. Many participants saw the task as being about using previous reasoning to answer interviewer questions. In this framing participants responded to interview questions by explaining phenomena using personal notions or theories based on previous reasoning. These ideas were based on multiple real world observations, as well as on formal instruction, or previous gaming experiences. In this framing knowledge is neither constructed on-the-spot, nor is it expert-like knowledge that is simply being recited without understanding. Examples of adopting a previous reasoning frame include Corey's attempt to describe how the movement of the car at a stop light depends on whether or not the car intends to turn left, right, or go straight, and John's references to geography's impact on the speed of a car. The three main framing codes and sub-codes can be seen below in Table 1.

Two participants, Brian and John (both considered themselves game designers), frequently engaged in on-the-spot construction of game stories throughout the interview. For these two participants, the interview was about constructing action-packed game-related stories to describe answers to interview questions. For example, when asked to describe the motion of a car after a red light turns green, Brian states:

Well I tried, I just kept moving as fast as I could to make sure no one else is coming after me. Maybe I may have used a turbo boost like a mushroom. And then I bumped into the obstacle, which was your arm. And then I got hurt, and then the other racers past me.

Throughout the interview Brian enacts many other racing situations, imagines gamerelated explanations for why a car might be driving slow, and even describes the difference between two speeds as being mostly about how one would animate the wheels. Brian's onthe-spot construction of game stories is a relatively stable frame that continues throughout the first two thirds of the interview. John, on the other hand, only occasionally draws on gaming experiences during the pre-game interview. While talking about a car keeping a constant pace John states this might be "boring." When asked to clarify, John suggests that a car might stay at 20 if there was a "rule" or if it was a "timed race." 


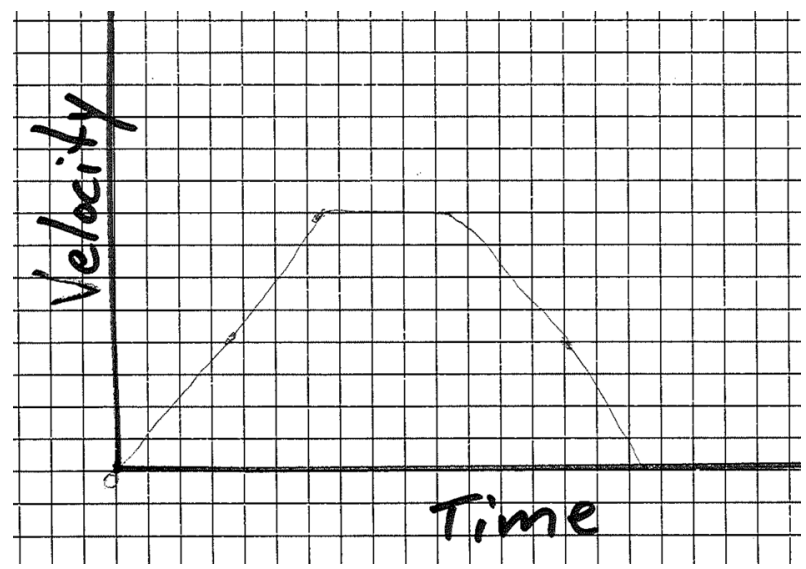

Fig. 7 John's pre-game graph was considered "qualitatively correct" because it is a line graph that properly utilizes both the $\mathrm{x}$ - and $\mathrm{y}$-axes. However, John's graph fails to show changes in acceleration

Table 1 Player epistemological framing coding scheme

\begin{tabular}{ll}
\hline $\begin{array}{l}\text { On-the-spot } \\
\text { construction }\end{array}$ & On-the-spot constrution of idea, theory, or story \\
\hline $\begin{array}{l}\text { Real world } \\
\text { Formal instruction }\end{array}$ & $\begin{array}{l}\text { "Waybe you alternate the gas and brake pedal to keep the needle from moving" } \\
\text { "Wame }\end{array}$ \\
$\begin{array}{l}\text { "I just kept moving as fast as I could to make sure no one else is coming after me, } \\
\text { maybe I use a turbo boost like a mushroom" }\end{array}$
\end{tabular}

Previous reasoning Drawing on previous reasoning or observations to explain

Real world "You have to push the pedal harder to go faster"
Formal instruction "It would mean the miles per hour. That's why they call it that-how much time it
takes to get this amount of miles"
"Like if it's a timed race? Like, most racing games are timed so you can compare
your scores"

Expert-like Reciting expert-like knowledge as "fact"

\begin{tabular}{|c|c|}
\hline Real world & $\begin{array}{l}\text { "If you step on the accelerator, more fuel gets injected, as more fuel gets injected } \\
\text { the engine works faster" }\end{array}$ \\
\hline Formal instruction & $\begin{array}{l}\text { "I forgot what type of graph it was, but it's a graph. It could be a bar graph, or line } \\
\text { graph, or something like that" }\end{array}$ \\
\hline Game & $\begin{array}{l}\text { "Well, the animation move faster for } 40 \text { miles, and move slower for } 20 \text { miles } \\
\text { per hour" }\end{array}$ \\
\hline
\end{tabular}

While participants were generally consistent in their epistemological frames in the first two thirds of the interview, many participants experienced clear epistemological mismatches when trying to make sense of the graphing task. The introduction of this formal representation in the interview contexts caused most participants to shift frames- to assume the task was now about showing expertise with memorized formal knowledge. When handed a pre-labeled piece of graphing paper, John quickly states, "I'm not sure 
what kind of graph it is, but it's a graph [...] it could be a bar graph, or um, what's it called? A line graph, or something like that." After the interviewer explained the task, John nervously asks, "what type of graph" and continues to question himself with statements like, "so usually you start at 0 , right?" and "it'd be like increasing?" Another participant, Walt, after receiving the graph paper but before the task is explained attempts to show his expertise and states, "It would normally be like that [shows a straight positively slanted line]." When questioned about what he means, Walt explains, "Sometimes it's just a right angle, but at other times, it can vary."

Brian, the "game designer" who playfully framed most of the interview as being about constructing stories of game action, experienced a very visible framing mismatch when handed the graphing paper. Just as Brian begins to construct the graph he becomes visibly uncomfortable, pauses, and for the next ten seconds mumbles, "Uh, wait. I'm trying to think which way to go. Lets see, the faster...the faster it moves, which means time....alright, ok, I'm ready." It's at this moment, when he begins to consider the graphing paper, that Brian's physical demeanor changes, as does his playful attitude. When asked to describe the graph he eventually creates, Brian states:

Brian: Let's see...so, since the y is the speed velocity, like miles per hour, uh...cars, the car increased as the plot, as the plotted points are moving more up. As long...the steeper the slope is the speed of the car.

Interviewer: The, the steeper the slope is the speed?

Brian: Yep.

Interviewer: Okay.

Brian: The speed rate.

When presented with game-related and real-world representations (such as the speedometer) during the pre-game interview, Brian frequently invokes a fictional car as it enacts game-like action. However, when confronted with the graphing paper, Brian shifts away from this on-the-spot game story framing to one that privileges expert-like formal knowledge. Brian demonstrates this shift as he struggles to use formally sanctioned vocabulary like "slope" as well as mixed terms such as "speed velocity" and "speed rate." While in this frame Brian does not see his expert gaming knowledge as relevant.

The results of the pre-game interview suggest that participants have a variety of intuitive knowledge to draw on when reasoning about kinematics. While all participants have extensive experience playing racing video games, few drew on these gaming experiences to answer motion-related questions. The two participants that did draw on gaming knowledge considered themselves both expert gamers as well as game designers and also struggled to integrate this gaming knowledge with more formal kinematic ideas.

The pre-game graphing task revealed that participants genuinely struggled to connect the car and speedometer to a formal graph representation. For most participants, the creation of a graph seemed at odds with the stories and descriptions utilized during the rest of the pre-game interview. John, Walt, and Brian's sudden use of formal vocabulary and their spontaneous attempts to list many different kinds of graphs indicate each engaged an epistemic frame that privileges expert-like knowledge. While in this frame, participants see the activity to be about reciting factual knowledge and as such, begin to draw almost exclusively on resources specific to formal learning contexts and neglect knowledge resources tied to intuition and gaming experiences. 


\subsection{Post-game Interviews and Graphing}

During the post-game interview, conducted about two to seven days after playing FTR and at least two weeks after the pre-game interview, participants showed improvements on graph construction despite the lack of direct instruction or reflection on in-game tasks and representations. In the post-game interview, five of the six participants created qualitatively correct velocity-versus-time graphs (Fig. 8). Three of these five verbally cited the game when beginning the graphing task in the post-game interview. The one participant that did not complete a "correct" graph in the post-game interview was the same participant that was unable to complete any graph in the pre-game interview. Interestingly enough, when handed the graphing paper in the post-game interview, even this participant stated, "I was confused on this the last time so [...] is it just like the graph we did in the game?"

All five of these graphs contained important features such as differing amounts of acceleration and moments of constant velocity. John, who had previously given a simple, almost literal description of his graph in the pre-game interview is much more nuanced when explaining his post-game graph. John states:

John: If, like, in a second, and you just accelerate like that, it will just go...roughly the same block [draws a line with a sharp slant in one column]...

Interviewer: So is that because I'm going to a higher number or is that because...

John: It's because of the time [pause] like it just keeps on increasing. It'd be, yeah, it would be more steep.

When asked about areas of his graph that showed varying degrees of acceleration, Mike, who had created a pencil-as-car graph in the pre-game interview, states, "You were going a lot faster and the velocity is going up [...] you kinda went up a bit slower and then stayed at 20, and then you got a little bit higher and stayed at 40." While Mike attends mostly to discrete velocities, he also attends directly to how quickly these values change, showing some mastery with the interdependency of these constructs.

Collin, the participant who did not create what we would call a qualitatively correct graph in the post-game interview, was still able to identify the shortcomings of the graph he did create. While Collin's graph utilizes both the $\mathrm{x}$ - and $\mathrm{y}$-axis, he did not consider "time" as an accumulating value. Instead, Collin counted out how long it took the interviewer to move the needle from one location to the next, moved that distance along the $\mathrm{x}$-axis, beginning at zero each time, and then moved up the y-axis to the final speed indicated on the speedometer. As he discusses his graph, Collin quickly notes that it does not match reality and states, "if you were driving a real car I don't know if it would take four seconds to get to 10 miles an hour." Collin also correctly identifies that his graph cannot account for areas of constant velocity or when the car decelerates to zero. He even goes on unprompted to tell the interviewer, "I was also doing it from where you stopped so only 10 and $20 \ldots$ so it...I wouldn't do like square after square." His observation that his graph does not deal adequately with the car's velocity in the midst of a change reveals that Collin has gained a significant meta-epistemological understanding of what his graph can and cannot communicate (diSessa et al. 1991).

In the pre-game interview, Brian enacted a game story epistemic frame that caused difficulty when reasoning about formal representations. During the post-game interview Brian once again answered many interview questions using video game stories: however, when describing the motion of two different toy cars being pushed by the interviewer across the table - one at constant velocity and the other with constant acceleration-Brian casually states, "Well the other one was just this constant rate, but what you did just now, 
(a)
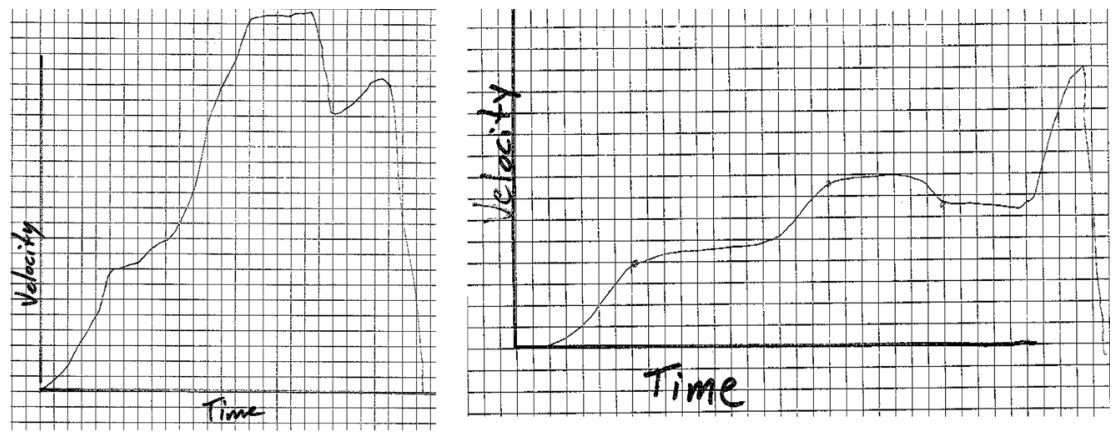

(b)
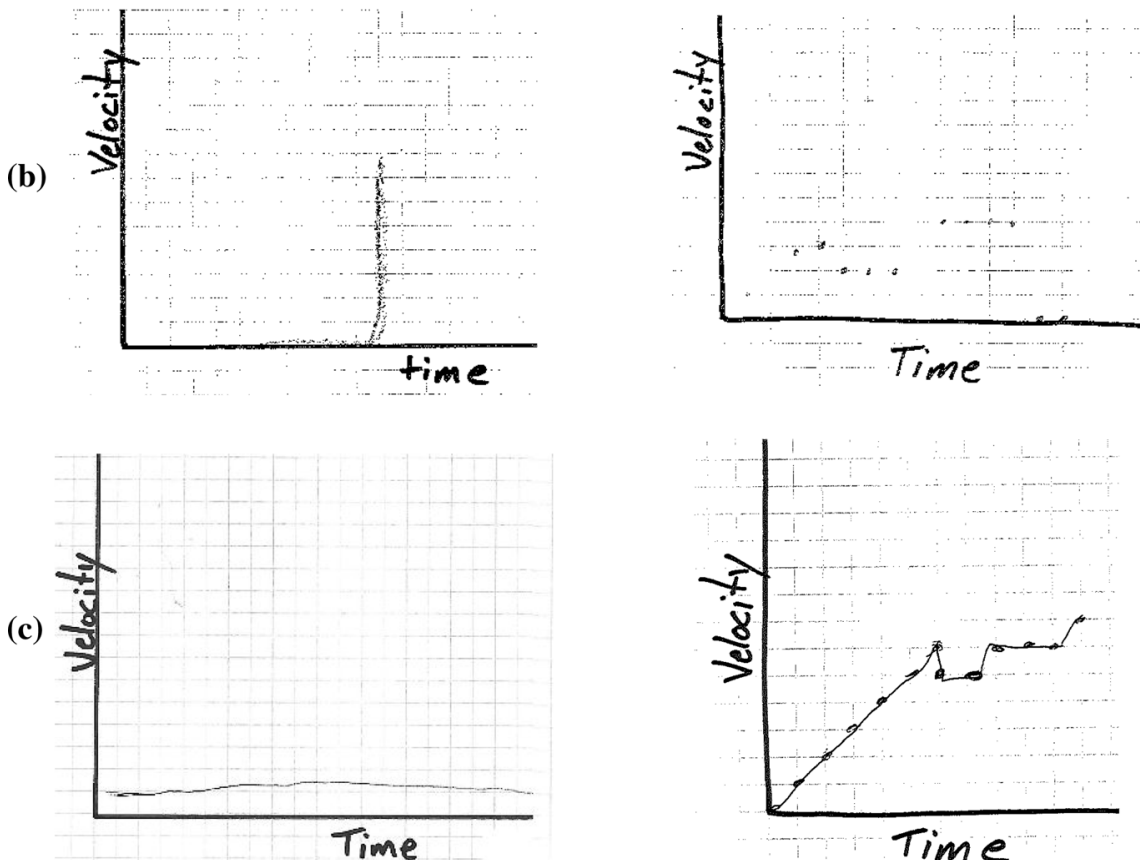

Pre-game

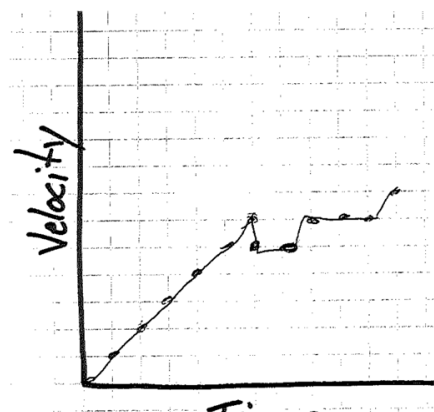

Time

Post-game

Fig. 8 Three examples of how pre- and post-game graphs changed. Brian (a) created a qualitatively correct graph in the pre-game interview; however, in the post-game interview Brian has added "points" to his graph. Corey (b) and Mike (c) both created pencil-as-car graphs in the pre-game interview, but created qualitatively correct graphs in the post-game interview that also included points

be where...where the slope would increase." Brian's spontaneous use of the term "slope," invoking a graph representation, is particularly interesting as no graphs have been used yet at this point in the post-game interview. When pushed by the interviewer to explain what he means by slope, Brian states, "Well that's what I learned in algebra." Later in the interview, when describing his post-game graph, Brian frequently intersperses formal vocabulary such as "constant rate," with more informal game-related terms like "boost." Of note is that Brian's use of this formal vocabulary is not marked by visible shifts in behavior or language patterns, as was the case in his pre-game interview. 
If the constructible authentic representations principle is responsible for the changes in player graphing and epistemic framing described above, one might expect in-game representations and features to find their way into post-game graphs. In three of the six graphs constructed, participants added points at each time step rather than simply drawing a line to represent the motion (examples of this can be seen in Fig. 8 above). One of these participants, Mike, initially created his graph using only dots, adding lines to then connect the dots once he was finished (Fig. 8b). When asked why he used dots to represent his graph, he stated simply, "Because the game had dots." Another participant, Brian, who made a "correct" graph that did not include points before playing the game, added them to his post-game graph while describing it to the interviewer (Fig. 8a). In any other circumstance, dots on a graph may not be very notable. In this case, however, there is a sharp continuity between post-game graphs constructed by players and the points players accelerate during the pit boss level of FTR. The fact that multiple participants cited these specific features during the post-game graphing task further suggests that players are drawing directly on this in-game representation to complete non-game tasks.

This point should not be overlooked. Players do not receive explicit instruction on graphing in the game; instead, they are simply confronted with a racing task and are encouraged to "figure it out." Furthermore, the tools (paper and pencil) and representations (speedometer) used in the interview protocol are completely absent from the game context. While players do ultimately create a graph in-game, these graphs are constructed by "racing" points along the y-axis using the roll of a motion-sensitive controller as described in the methods section, and no directions are provided on what constitutes a "correct" graph. The ability of participants to draw on these "graph construction" resources outside of the game context with different tools and representations is exciting, considering the large body of work that suggests transferring such skills or knowledge to a different context is an unlikely occurrence (Gentner et al. 2003; Gick and Holyoak 1980, 1983; Mestre 2002).

\section{Discussion}

We began this paper by framing two major challenges: designing educational video games that align with youth culture, and creating gaming experiences that can be utilized by players when thinking and reasoning in non-game contexts. While we have modest evidence to suggest that players enjoyed the game and did play on their own after the experiment concluded, this paper primarily addressed the second problem. To that end, we proposed the principle of constructible authentic representations and presented data from a study of players interacting with a racing game designed to incorporate this principle.

Participants showed a remarkable change in graphing ability after playing FTR. Many participants struggled in the pre-game graphing task with only three of eleven participants creating qualitatively correct graphs. Two of these three completing graphs that included areas of constant velocity and changing acceleration with the other creating a correct line graph that lacked some of these important features. Nonetheless, these three participants clearly had at least a basic understanding of the velocity versus time graph before playing FTR. In the post-game graphing task nearly all participants (five of the six that played the game and completed the post-game interview) were able to create qualitatively correct graphs despite the lack of any explicit instruction on graphing. Of the three participants that had failed to create a qualitatively correct graph in the pre-game interview two of these had completed a pencil-as-car graph and one was unable to create any kind of graph. In the 
post game interview all three completed complex graphs that exhibited a rich understanding of relevant kinematic concepts such as changing acceleration (Table 2).

In addition to successfully constructing velocity versus time graphs in the post-game interview, players also added graphical features to post-game graphs there were congruent to features found in the graphing phase of FTR. Two of the three participants that added points to their post-game graphs created pencil-as-car graphs in the pre-game interview. The third participant had completed a "correct" graph in the pre-game interview but added points to his post-game graph while explaining graph features to the interviewer. All three participants that struggled with the graphing task in the pre-game interview cited the game during graph construction in the post-game interview. The success of post-game graphing and the addition of in-game graphing features suggest that the design of FTR encourages players to see experiences and constructions in-game as relevant in settings beyond the game. But if players didn't see previous gaming experiences as relevant in the pre-game interview, how is it that FTR experiences are seen as relevant in the post-game interview?

One might argue that the game and interview contexts are not sufficiently different. This argument would likely hinge on the belief that because the interviewer was the same in all meetings, participants perceived the contexts to be the same. We find this to be an inadequate explanation for the results presented in this study as a large body of work exists that shows a dismal record of transfer on tasks and context much more aligned than the one presented here (Gentner et al. 2003; Gick and Holyoak 1980, 1983). Furthermore, the data presented of player epistemic mismatches and dramatic framing shifts during the interview indicates players perceive the game and interview contexts as separate (indeed, in some cases we saw multiple shifts during the same interview).

Nevertheless, we contend that participants showed success on the post-game graphing task because they both saw in-game experiences as relevant to reasoning about these formal representations and real-world phenomena and because they came to appreciate the ability of these formal representations to help them to achieve a personally meaningful goal-namely winning the game. In FTR game mechanics were designed to be conceptually integrated - to map directly onto the concepts of interest. Because winning the game means successfully navigating and manipulating game mechanics, such a design ensures that players are constantly exploring and interacting with the boundaries and connections between target concepts as they strive to master game mechanics and achieve in-game success. In FTR players are encouraged to explore the relationship between acceleration and velocity through interactions with two key game mechanics: a motion-sensitive controller and track painting. The motion-sensitive controller foregrounds ideas of acceleration, allowing players to map the embodied sense of "speeding up" and "slowing down" to actions enacted by the car. On the other hand, track painting foregrounds velocity. Here players begin to connect those embodied notions of acceleration to discrete values of velocity and track features. Both representations encode the fundamental conceptual structure of the target domain (Cheng 2011, p. 476).

While the development of these knowledge structures is important, it means very little if players do not see these experiences and resources as relevant when engaging in reasoning outside of the game. While all players had many racing video game experiences before playing FTR, most did not draw on these experiences when answering questions in the pregame interview. Only two participants seemed to use game experiences extensively in the pre-game interview. Not surprisingly these participants identified themselves strongly as expert gamers and game designers. This personal identity, and because the study was presented to participants as a "games study," likely encouraged these participants to see this game knowledge as relevant. Unfortunately even these participants struggled to juggle 
Table 2 Pre- and post-game graphs created by players

\begin{tabular}{lrllcc}
\hline Participant & Age & Pre-game graph & Post-game graph & Dots? & $\begin{array}{l}\text { Cited } \\
\text { game? }\end{array}$ \\
\hline Corey & 8 & Pencil-as-car & Line graph with all features & Yes & Yes \\
Mike & 12 & Pencil-as-car & Line graph with all features & Yes & Yes \\
Collin & 9 & None & Acceleration bar chart & No & Yes \\
Walt & 11 & Line graph with all features & Line graph with all features & No & No \\
John & 13 & Line graph without changing acc & Line graph with all features & No & No \\
Brian & 14 & Line graph with all features & Line graph with all features & Yes & No \\
\hline
\end{tabular}

an epistemological frame that privileges game knowledge and one where formal school knowledge is paramount as frame shifting was visibly difficult during the pre-game interview.

In contrast, during the post-game interview multiple participants drew on FTR game experiences when answering kinematic questions. It is possible this is due to the use of constructible authentic representations throughout the design of FTR. This design connects knowledge structures developed and/or refined in-game to key tools and representations important in the target domain. In FTR, this is accomplished by correlating racing mechanics and construction tools in the pit boss level to formal velocity-versus-time graphs. Throughout the game, a velocity-versus-time graph acts as a stand-in for a speedometer while the player drives the car around the track with the motion sensitive controller. In the pit boss level, gameplay suddenly shifts when players are faced with the challenge of driving the car around the track by constructing a plan rather than racing. Constructions in the pit boss level include track painting as well as graph building. To construct the graph, players accelerate points up and down a y-axis using the motion sensitive controller.

By reusing the driving mechanic players have become familiar with throughout the game, the racing experience is directly tied to graph construction. This enables the players to connect the sense of speeding up and slowing down that they have become familiar with during the racing phase to important graph features such as instances of positive and negative acceleration. Because a complete graph is constructed before the car drives around the track, players must also connect these graph features to segments of the track, such as straightaways and corners.

Playing FTR provides players with a rich and useful set of resources with which to reason about kinematics in-game. Yet more importantly, we see evidence of players using these resources when reasoning about non-game kinematic phenomena. Because the graph representation constructed in-game (which, as we have just discussed is highly connected to the other key in-game experiences) is also an authentic representations, one recognizable outside of the game space and central to formal education experiences, players are more likely to see the processes and primitives used to construct the representation in-game as relevant and "real." Furthermore, because graph construction becomes a powerful and effective tool to "win the game," players can begin to see this previous "formal" representation as one that can help them to accomplish personally meaningful goals.

There are two possible mechanisms that may be at work here. While we specifically chose young participants for our study, it is possible that some players have already acquired a relatively rich understanding of this particular graph representation. In this case, 
as in-game experiences become linked to the graph, these experiences also become linked to other non-game resources the player has already acquired and connected to the graph representation. This seems to be the case with the older participants as most recognized the graph representation, and some were able to construct something resembling a velocity versus time graph in the pre-game interview. While some of these pre-game graphs lacked important features such as areas of constant velocity and varying degrees of acceleration, these participants held basic knowledge of formal kinematic constructs as well as some intuitive knowledge about motion. However, as previously indicated, before playing FTR these players tended to separate motion intuition, game-related experiences, and knowledge resources acquired in more formal learning settings. For these players, interactions with FTR's CAR design allows them use intuitions about motion to make sense of previously acquired formal terms, concepts, and representations leading to a tighter coupling of these previously unconnected knowledge resources.

However, some participants had little experience with graphs. Here, an understanding of this representation and its relationship to concepts such as acceleration and velocity may be developed while playing the game. In this case, it is not until players are confronted with the graph representation outside of the game that these resources would be seen as relevant to non-game problem solving. In either case, once this common representation has become connected to both in-game experiences as well as more formal math and physics instruction, knowledge resources that have developed alongside this representation would also become connected. In future work it will be important to more precisely identify the role prior knowledge plays in the co-activation of game and domain-specific resources.

In the post-game interviews students demonstrated an ability to more fluidly discuss game experiences and stories as well as formal representations and terminology. This is in sharp contrast to the halting epistemic shifts that occurred in the pre-game interview when confronted with the graphing task. We see this as evidence of FTR players enacting a different epistemic frame (or set of frames) during the post-game interview. Exactly what form this new epistemic frame takes remains unclear.

Players may be enacting a new, hybrid frame that combines both formal and game knowledge. In this frame, players see experiences and representations found in-game, as well as formal concepts and representations related to these in-game experiences, as relevant during the interview. However, it is unclear whether or not this hybrid frame would also be activated during formal instruction.

Alternatively, players may still be engaging in frame shifts during the interview, but these shifts would be occurring much more fluidly. In this case, the shift is facilitated by the common representation (in this case, the graph), so that when players activate a formal epistemic frame during the interview, game knowledge is also seen as relevant, regardless of the context in which it was learned. If this is in fact what was occurring during the study, then it is likely that game experience would also be seen as relevant in more formal contexts when the bridging representation is utilized.

In Sect. 1.2 we acknowledged the challenge of creating CAR games for all imaginable STEM content areas. While it is certainly more difficult to design an educational game to include conceptually integrated mechanics and visually and epistemologically aligned representations, the findings of this paper suggest the result is worth the effort. To increase our confidence in the robustness of the CAR principle, we have designed and tested an additional prototype game for an alternate game genre and content domain. ${ }^{3}$ The success of this second

\footnotetext{
${ }^{3}$ We provided a brief description of how this second prototype game, Particles!, incorporates the CAR principle in Sect. 1.2.
} 
prototype, which is discussed in more detail elsewhere (Holbert 2013), suggests that the CAR principle may be effective in a wide range of game types and STEM domains.

\section{Conclusion}

In this paper we have outlined a new design principle, constructible authentic representations, for designing educational video games experiences that activate in the player a set of knowledge resources that make it likely that these resources will get activated again in a non-game contexts. CAR games are construction games where the building primitives that are designed to integrate the concept of interest are used to construct artifacts that are epistemologically and visually aligned to representations heavily utilized in the target domain. We argue that games designed in this way encourage players to connect in-game experiences (which match the concepts of interest) to representations that have meaning outside of the game space and we described a study of six children playing a game of our design to that effect.

These results increase our optimism that informal video game play can be a powerful space for the exploration of science phenomena. Because games that utilize a constructible authentic representations design avoid a didactic approach, such games are more likely to survive in the diverse gaming ecology that exists outside of the classroom. Furthermore, approaching educational game design from an epistemic framing perspective allows us to see more clearly a set of solutions that go beyond simply making the content more explicit. Because CAR games can serve both as engaging and entertaining play spaces as well as effective spaces for practicing and enacting scientific reasoning, it follows that the design of both commercial and educational games would benefit greatly from incorporating this design principle.

By carefully choosing interaction mechanisms and primitives for the construction component of video games, and by ensuring the final constructions will be seen as relevant to contexts beyond the video game, game-related knowledge resources created and refined by interactions with such games are then free to be utilized in non-game settings. By including constructible authentic representations in popular commercial games, it just might be possible to make Mario as useful as the Turtle.

Acknowledgments The authors would like to thank Bruce Sherin, Reed Stevens, Rosemary Russ, Corey Brady, Michael Horn, Pryce Davis, David Weintrop, and all members of the CCL for their insightful comments on this work.

\section{References}

Annetta, L. A. (2008). Video games in education: Why they should be used and how they are being used. Theory into Practice, 47(3), 229-239.

Bamberger, J. (1996). Turning music theory on its ear: Do we hear what we see; do we see what we say? International Journal of Computers for Mathematical Learning, 1, 33-55.

Barab, S., Sadler, T. D., Heiselt, C., Hickey, D., \& Zuiker, S. (2007a). Relating narrative, inquiry, and inscriptions: Supporting consequential play. Journal of Science Education and Technology, 16(1), 59-82.

Barab, S., Thomas, M., Dodge, T., Carteaux, R., \& Tuzun, H. (2005). Making learning fun: Quest Atlantis, a game without guns. Educational Technology Research and Development, 53, 86-107.

Barab, S., Zuiker, S., Warren, S., Hickey, D., Ingram-Goble, A., Kwon, E., et al. (2007b). Situationally embodied curriculum: Relating formalisms and contexts. Science Education, 91(5), 750-782. doi:10. 1002/sce.20217. 
Barendregt, W., \& Bekker, T. M. (2011). The influence of the level of free-choice learning activities on the use of an educational computer game. Computers \& Education, 56(1), 80-90. doi:10.1016/j.compedu. 2010.08.018.

Behesthi, E., Jona, K., Horn, M., Trouille, L., Weintrop, D., \& Wilensky, U. (2013). The physics of angry birds. CT-STEM Group. http://ct-stem.northwestern.edu/lesson-plans.

Boucher-Genesse, F., Riopel, M., \& Potvin, P. (2011). Research results for Mecanika, a game to learn Newtonian concepts. In C. Steinkuhler, C. Martin, \& A. Ochsner (Eds.), Proceedings of the 7th Annual Games, Learning, and Society Conference. Madison, WI.

Brofenbrenner, U. (1979). The ecology of human development: Experiment by nature and design. Cambridge, MA: Harvard University Press.

Cheng, P. C.-H. (2011). Probably good diagrams for learning: Representational epistemic recodification of probability theory. Topics in Cognitive Science, 3(3), 475-498.

Clark, D. B., \& Martinez-Garza, M. (2012). Prediction and explanation as design mechanics in conceptually integrated digital games to help players articulate the tacit understandings they build through game play. In C. Steinkuhler, K. Squire, \& S. Barab (Eds.), Games, learning, and society: Learning and meaning in the digital age. Cambridge: Cambridge University Press.

Clark, D. B., Nelson, B., Chang, H., Martinez-Garza, M., Slack, K., \& D’Angelo, C. M. (2011). Exploring Newtonian mechanics in a conceptually-integrated digital game: Comparisons of learning and affective outcomes for students in Taiwan and the United States. Computers \& Education, 57, 2178-2195.

Clark, D. B., Nelson, B., D’Angelo, C. M., Slack, K., \& Menekse, M. (2010). SURGE: Assessing students' intuitive and formalized understandings about kinematics and Newtonian mechanics through immersive game play. Presented at the Annual Meeting of the American Educational Research Association, Denver, CO.

Collins, A. (1995). Design issues for learning environments. In S. Vosniadou, E. de Corte, H. Mandle, \& G. Robert (Eds.), International perspectives on the psychological foundations of technology-based learning environments (pp. 347-361). Hillsdale, NJ: Lawrence Erlbaum.

Cooper, S., Dann, W., \& Pausch, R. (2000). Alice: A 3-D tool for introductory programming concepts. Journal of Computing Sciences in Colleges, 15, 107-116.

diSessa, A. A. (1993). Toward an epistemology of physics. Cognition and Instruction, 10, 105-225.

diSessa, A. A., Hammer, D., Sherin, B., \& Kolpakowski, T. (1991). Inventing graphing; Meta-representational expertise in children. Journal of Mathematical Behavior, 10, 117-160.

Gee, J. P. (2003). What video games have to teach us about learning and literacy. New York: Palgrave Macmillan.

Gee, J. P. (2007). Good video games and good learning. New York, NY: Peter Lang.

Gentner, D., Loewenstein, J., \& Thompson, L. (2003). Learning and transfer: A general role for analogical encoding. Journal of Educational Psychology, 95, 393-408.

Gick, M. L., \& Holyoak, K. J. (1980). Analogical problem-solving. Cognitive Psychology, 12, 306-355.

Gick, M. L., \& Holyoak, K. J. (1983). Schema induction and analogical transfer. Cognitive Psychology, 15, 1-38.

Ginsberg, H. P. (1997). Entering the child's mind: The clinical interview in psychological research and practice. New York: Cambridge University Press.

Habgood, J. M. P., \& Ainsworth, S. E. (2011). Motivating children to learn effectively: Exploring the value of intrinsic integration in educational games. Journal of the Learning Sciences, 20, 169-206.

Hammer, D., \& Elby, A. (2002). On the form of personal epistemology. In B. K. Hofer \& P. R. Pintrich (Eds.), Personal epistemology: The psychology of beliefs and knowledge and knowing (pp. 169-190). Mahwah, NJ: Erlbaum.

Hammer, D., Elby, A., Scherr, R. E., \& Redish, E. F. (2005). Resources, framing, and transfer. In J. Mestre (Ed.), Transfer of learning from a modern multidisciplinary perspective (pp. 89-120). Greenwich, CT: Information Age Publishing.

Holbert, N. (2009). Learning Newton while crashing cars. Presented at the Games, Learning, and Society 5.0, Madison, WI.

Holbert, N. (2013). Reimagining game design (RiGD): Exploring the design of constructible representations for science reasoning (doctoral dissertation). Evanston, IL: Northwestern University.

Holbert, N., \& Wilensky, U. (2010a). FormulaT racing. Evanston, IL: Center for Connected Learning and Computer-based Modeling.

Holbert, N., \& Wilensky, U. (2010b). FormulaT racing: Combining gaming culture and intuitive sense of mechanism for video game design. In K. Gomez \& J. Radinsky (Eds.), Proceedings of the 9th International Conference of the Learning Sciences. Chicago, IL.

Holbert, N., \& Wilensky, U. (2011). FormulaT racing: Designing a game for kinematic exploration and computational thinking. In C. Steinkuhler, C. Martin, \& A. Ochsner (Eds.), Proceedings of 7th Annual Games, Learning, and Society Conference. Madison, WI. 
Holbert, N., \& Wilensky, U. (2012). Particles!. Evanston, IL: Center for Connected Learning and Computer-based Modeling.

Itō, M. (2008). Education vs. entertainment: A cultural history of children's software. In K. Salen (Ed.), The ecology of games: Connecting youth, games, and learning (pp. 89-116). Cambridge, MA: MIT Press.

Itō, M. (2010). Hanging out, messing around, and geeking out: Kids living and learning with new media. Cambridge, MA: MIT Press.

Kafai, Y. B. (1996). Learning design by making games: Children's development of design strategies in the creation of a complex computational artifact. In Y. B. Kafai \& M. Resnick (Eds.), Constructionism in practice: Designing, thinking, and learning in a digital world. Mahwah, NJ: Lawrence Erlbaum.

Kelleher, C., \& Pausch, R. (2006). Lessons learned from designing a programming system to support middle school girls creating animated stories. In J. Grundy \& J. Howse (Eds.), IEEE symposium on visual languages and human-centric computing (pp. 165-172). Brighton, UK.

Ketelhut, D. J., Clarke, J., \& Nelson, B. C. (2010). The development of river city, a multi-user virtual environment-based scientific inquiry curriculum: Historical and design evolutions. In M. J. Jacobson \& P. Reimann (Eds.), Designs for learning environments of the future (pp. 89-110). US: Springer.

Lenhart, A., Kahne, J., Middaugh, E., Macgill, A. R., Evans, C., \& Vitak, J. (2008). Teens, video games, and civics. PEW Internet \& American Life Project.

Levy, S. T., \& Wilensky, U. (2009). Students' learning with the connected chemistry (CC1) curriculum: Navigating the complexities of the particulate world. Journal of Science Education and Technology, $18,243-254$.

Mestre, Jose. (2002). Transfer of learning: Issues and research agenda. Arlington, VA: National Science Foundation.

Minsky, M. (1986). The society of mind. New York: Simon \& Schuster.

Nemirovsky, R., Tierney, C., \& Wright, T. (1998). Body motion and graphing. Cognition and Instruction, $16,119-172$.

Noss, R., \& Hoyles, C. (1996). Windows on mathematical meanings: Learning cultures and computers. Dordrecht: Kluwer Academic Press.

Papastergiou, M. (2009). Digital game-based learning in high school computer science education: Impact on educational effectiveness and student motivation. Computers \& Education, 52(1), 1-12.

Papert, S. (1980). Mindstorms: Children, computers and powerful ideas. New York: Basic Books.

Papert, S. (2000). What's the big idea? Toward a pedagogy of idea power. IBM Systems Journal, 39(3.4), $720-729$.

Papert, S., \& Harel, I. (1991). Situating constructionism. In S. Papert \& I. Harel (Eds.), Constructionism. New York: Ablex Publishing.

Piaget, J. (1929). The child's conception of the world. London: Rowman \& Littlefield.

Pratt, D., \& Noss, R. (2010). Designing for mathematical abstraction. International Journal of Computers for Mathematical Learning, 15(2), 81-97.

Resnick, M., Maloney, J., Monroy-Hernández, A., Rusk, N., Eastmond, E., Brennan, K., et al. (2009). Scratch: Programming for all. Communications of the ACM, 52(11), 60-67.

Robertson, J., \& Howells, C. (2008). Computer game design: Opportunities for successful learning. Computers \& Education, 50(2), 559-578. doi:10.1016/j.compedu.2007.09.020.

Roschelle, J., Kaput, J. J., \& Stroup, W. (2000). SimCalc: Accelerating students' engagement with the mathematics of change. In M. J. Jacobson \& R. B. Kozma (Eds.), Innovations in science and mathematics education: Advanced designs for technologies of learning (pp. 47-76). Hillsdale, NJ: Earlbaum.

Russ, R. S., Lee, V. R., \& Sherin, B. L. (2012). Framing in cognitive clinical interviews about intuitive science knowledge: Dynamic student understandings of the discourse interaction. Science Education, 96(4), 573-599. doi:10.1002/sce.21014.

Sherin, B. (2000). How students invent representations of motion: A genetic account. Journal of Mathematical Behavior, 19, 399-441.

Squire, K. (2006). From content to context: Videogames as designed experience. Educational Researcher, 35, 19-29.

Squire, K., \& Barab, S. (2004). Replaying history: Engaging urban underserved students in learning world history through computer simulation games. In Proceedings of the 6th International Conference on the Learning Sciences (pp. 505-512).

Tasar, M. H. (2010). What part of the concept of acceleration is difficult to understand: The mathematics, the physics, or both? ZDM, 42, 469-482.

The Alice Team. (n.d.). Alice. What is Alice? http://www.alice.org/index.php?page=what_is_alice/what_is_ alice. 
Trowbridge, D. E. (1989). Graphs and tracks: An application of manipulable graphics. Academic Computing, May, 24-25.

Trowbridge, D. E., \& McDermott, L. C. (1981). Investigation of student understanding of the concept of acceleration in one dimension. American Journal of Physics, 49, 242-253.

Valve Corporation. (2013). Teach with portals. http://www.teachwithportals.com.

Wilensky, U. (1991). Abstract meditations on the concrete and concrete implications for mathematics education. In I. Harel \& S. Papert (Eds.), Constructionism. Norwood, NJ: Ablex Publishing Corp.

Wilensky, U., \& Reisman, K. (2006). Thinking like a wolf, a sheep, or a firefly: Learning biology through constructing and testing computational theories-and embodied modeling approach. Cognition and Instruction, 24, 171-209.

Wilkerson-Jerde, M. H., \& Wilensky, U. (2010). Restructuring change, interpreting changes: The deltatick modeling and analysis toolkit. In J. E. Clayson (Ed.), Proceedings of constructionism 2010. Paris, France. 